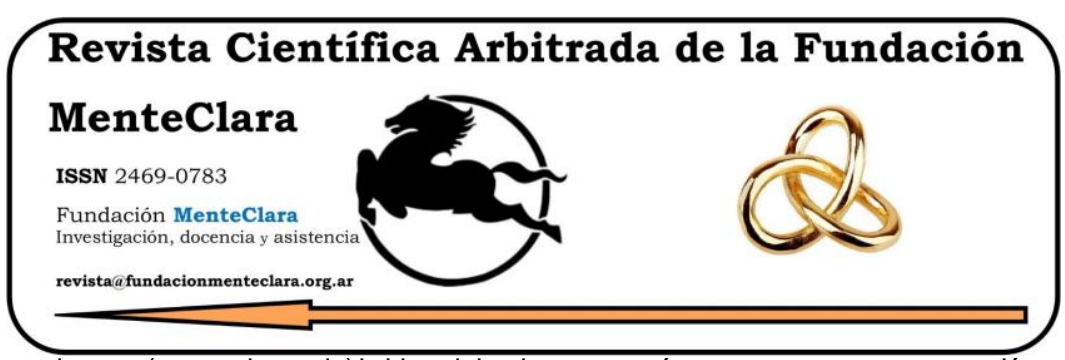

Artículos atravesados por (o cuestionando) la idea del sujeto -y su género- como una construcción psicobiológica de la cultura. Articles driven by (or questioning) the idea of the subject -and their gender- as a cultural psychobiological construction.

Vol. 6 (2021), enero-diciembre ISSN 2469-0783

https: / / datahub.io/ dataset/2021-6-e252

\title{
REPRESENTACIONES SOCIALES SOBRE LAS VACUNAS Y LA VACUNACIÓN FRENTE AL COVID 19
}

\author{
SOCIAL REPRESENTATIONS ON VACCINES AND VACCINATION AGAINST COVID \\ 19
}

Altamirano V.F., Bacon S.L., Baró S., Benitez D. A., Caravello J. C., Filippa N. L., Gastaldo Z. G., Gómez Jiménez C., Grañana N. E., Lavoie K. L., Losada A. V., Medina P. I., Muñoz M. A., Nogueira Da Silva A. C., Peláez S., Pérez A. R., Potes M. V., Santojanni L. B., Sorbara S. E., Souza Godinho S., Taliercio A. E. \& Zaracho, R. J. (1)

Cómo citar este artículo / Citation: Altamirano V.F., Bacon S.L., Baró S., Benitez D. A., Caravello J. C., Filippa N. L., Gastaldo Z. G., Gómez Jiménez C., Grañana N. E., Lavoie K. L., Losada A. V., Medina P. I., Muñoz M. A., Nogueira Da Silva A. C., Peláez S., Pérez A. R., Potes M. V., Santojanni L. B., Sorbara S. E., Souza Godinho S., Taliercio A. E. \& Zaracho, R. J. (2021). Repre sentaciones Sociales sobre las Vacunas y la Vacunación fre nte al COVID 19. Revista Cientifica Arbitrada de la Fundación MenteClara, Vol. 6 (252). DOI: https: / / doi.org/ 10.32351/rca.v6.252

Copyright: (C) 2021 RCAFMC. Este artículo de acceso abierto es distribuido bajo los términos de la licencia Creative Commons Attribution 4.0 International License (CC BY 4.0). Recibido: 30/08/2021. Aceptado: 14/09/2021 Publicación online: 16/09/2021

Conflicto de intereses: Ninguno que declarar.

Fuente de financiación: Montreal Behavioural Medicine Centre y Universidad de Flores. La presente investigación forma parte del estudio multicéntrico Awareness and Responses Evaluation Study (iCARE).

\section{Resumen}

Las representaciones son descriptas como un estilo específico de conocimiento, un modo determinado de descifrar la realidad, aludiendo al pensamiento del sentido común, siendo asociadas a un desarrollo personal que supone una ordenación cognitiva y a la vez halla su inicio, su difusión y cambio en el contexto sociocultural. En el contexto sanitario dado a causa de la pandemia por COVID 19 y mostrando como la mayor estrategia empleada por los diferentes gobiernos del mundo la vacunación toma relevancia el conocimiento sobre las representaciones acerca de la 
vacunación y las vacunas. En el marco del estudio multicéntrico International COVID 19 Awareness and Responses Evaluation Study (ICARE) liderado por Montreal Behavioural Medicine Centre se desarrolló una encuesta con una muestra 3330 adultos argentinos, de entre 18 y 70 años compuesta mayoritariamente por estudiantes y profesionales universitarios. El objetivo de la investigación ha sido conocer las representaciones acerca de las vacunas y la vacunación. Entre los resultados se destacan las representaciones sociales de los participantes que exhiben una gran adherencia a la vacunación y a las vacunas, con conceptos positivos en 8 de cada de 10 y un claro sostenimiento de las medidas de protección de como aislamiento y uso de más carilla acompañando la inoculación mientras que se conviva con el COVID 19.

\section{Abstract}

The representations are described as a specific style of knowledge, a specific way of deciphering reality, alluding to common sense thinking, being associated with a personal development that involves cognitive ordering and at the same time finds its beginning, its diffusion and change in the sociocultural context. In the health context given by the COVID 19 pandemic and showing how the greatest strategy used by the different governments of the world, vaccination takes relevance knowledge about representations about vaccination and vaccines. Within the framework of the multicenter study International COVID 19 Awareness and Responses Evaluation Study (ICARE) led by Montreal Behavioral Medicine Center, a survey was developed with a sample of 3330 Argentine adults, between 18 and 70 years old, composed mainly of university students and professionals. The objective of the research has been to know the representations about vaccines and vaccination. Among the results, the social representations of the participants that show great adherence to vaccination and vaccines stand out, with positive concepts in 8 out of 10 and a clear support for protection measures such as isolation and use of more veneer accompanying inoculation while living with COVID 19.

Palabras Claves: Representaciones sociales; Vacunas; Vacunación; COVID 19

Keyw ords: Social representations; Vaccines; Vaccination; COVID 19 


\section{Introducción}

Las representaciones sociales han sido postuladas por Moscovici como un conjunto ordenado de conocimientos en una actividad psíquica a partir de la cual los seres humanos permiten que sea clara la realidad física y social, ya que, se incluyen en un grupo o vinculo periódico de intercambios en el cual sueltan el vigor de su imaginación. Las representaciones se presentan como un estilo específico de conocimiento, un modo determinado de descifrar la realidad, aludiendo al pensamiento del sentido común. Las representaciones sociales responden a un desarrollo personal que supone una ordenación cognitiva y a la vez halla su inicio, su difusión y cambio en el contexto sociocultural. En esta dinámica toma significado la relación existente entre las representaciones sociales y la comunicación, ya que esta construcción se presenta en el acontecer diario y bajo una pluralidad de estímulos (Rivela y Losada, 2021).

En relación al contexto sanitario el estudio de Bacon et al. (2021) postulo entre los hallazgos una preocupación central en el $80 \%$ de los participantes de que un miembro de la familia no conviviente o allegados se infectará de COVID19. Las respuestas de la pesquisa exhibían mayor desazón por los impactos potenciales del COVID-19 en otras personas cercanas que en sí mismos. Estos hallazgos podrían dar cuenta de conductas de solidaridad y prosociales muy relevantes a la hora de diseñar campañas de prevención y desarrollo estratégico de políticas públicas, fomentando y haciendo foco en el cuidado del otro.

En el marco de la emergencia sanitaria producto de la pandemia por COVID 19, se sancionó la Ley 27.573 (2020) que declara "de interés público la investigación, desarrollo, fabricación y adquisición de las vacunas destinadas a generar inmunidad adquirida contra la COVID-19". Esta ley faculta al Poder Ejecutivo Nacional, a realizar los trámites 
necesarios para la adquisición de vacunas contra el Coronavirus. En Argentina el plan de vacunación inició a finales del año 2020, para el 1 de enero de 2021 habían sido inoculadas 121 personas con la vacuna Sputnik V 1er componente. Al momento del cierre de la encuesta analizada, se habían aplicado 367.335 dosis (Ministerio de Salud, 2021).

Las vacunas aplicadas hasta el momento de obtención de los datos de la presente investigación fueron Sputnik V, Covishield, Sinopharm y AstraZeneca; todas autorizadas para mayores de 18 años con modalidad de doble dosis (Ministerio de Salud, 2021). Se estableció un Plan Estratégico de Vacunación que determinó un esquema de priorización para la organización de la inoculación de la población objetivo (Ministerio de Salud, 2019). El Estado define que proveerá las vacunas para todos los que integren la población objetivo, independientemente de la cobertura sanitaria, el proceso será en etapas, voluntaria e independiente del antecedente de haber padecido la enfermedad.

Acompañando el proceso de vacunación, se difundieron por los medios de comunicación masiva una serie de noticias falsa (Fake news) poniendo en duda la eficacia y la seguridad de las vacunas. En el mes de junio de 2021 en un programa de televisión, un profesional de la salud afirmó que “toda vacuna, para ser éticamente aprobable, debe tener entre el 90 y el 95\% de efectividad" (Télam, 2021). Además, se afirmó en el mismo medio que para el caso de las vacunas contra COVID-19, no era suficiente la cantidad de pacientes con quienes se realizaron los ensayos clinicos para probar la efectividad. También se difundió en diversas notas de medios digitales, que seis voluntarios en los ensayos de Fase III de la vacuna de Pfizer-BioNTech murieron, sugiriendo que dichos decesos fueron consecuencia de la vacuna. (Télam, 2021); noticia desmentida en medios oficiales. 
El objetivo de la presente investigación ha sido conocer las representaciones sociales de una muestra argentina acerca de las vacunas y la vacunación.

\section{Método}

En el marco del estudio multicéntrico International COVID 19 Awareness and Responses Evaluation Study (ICARE) liderado por Montreal Behavioural Medicine Centre se llevó adelante una encuesta online auto administrable, participando de la misma 3330 adultos argentinos, de entre 18 y 70 años. El Estudio ICARE asociado a las políticas de salud pública busca conocer si es loable optimizar las estrategias aplanando la curva de infección por COVID-19 (Bacon et al., 2020).

La administración se llevó adelante durante mayo y junio de 2021. La difusión de la misma se realizó a través de redes sociales afines al equipo de investigación.

La muestra se constituyó por nivel educativo completo en un 1,8\% $(n=6)$ de participantes con estudios primarios, 18,6\% estudios secundarios $(\mathrm{n}=620), 11,7 \% \quad(\mathrm{n}=390)$ nivel educativo terciario, universitario $36,3 \%(n=1210)$ y con posgrado $36 \%(n=1200)$. Se advierte una activa participación de sujetos con altos niveles formativos.

En cuanto a la situación laboral de los encuestados puede precisarse que los estudiantes representaron al 17,4\% ( $\mathrm{n}=580)$, empleados de medio tiempo $14,7 \%(n=490)$, empleados de tiempo completo $35,7 \%(n=1190)$, trabajadores autónomos y profesionales independientes 44,4\% ( $n=1480)$, jubilados $5,1 \%(n=1709)$ y desempleados en $2,4 \%(n=80)$.

La participación en encuestas online se asocia en su mayoría a profesionales, estudiantes y sujetos de nivel educativos altos, 
estimándose esta coincidencia en la pesquisa dados los recursos afines a los miembros del equipo que desarrollo la investigación.

Bajo los principios éticos de la investigación se administró el instrumento de Consentimiento Informado. Este recurso se constituye como una herramienta que clarifica la participación, el grado de implicancia y reserva de los datos (Losada, 2014).

\section{Resultados}

Al respecto de haber recibido el esquema de vacunación en forma completa es decir con ambas dosis el 48,9\% respondió afirmativamente.

Ante la consulta de en qué medida influye en su decisión de vacunación los factores de:

- Conveniencia de recibir la vacuna en términos de aplicación, cercanía y gratuidad.

- Recibir la recomendación de mi empleador y/o autoridad laboral para vacunarme.

- Tener información de que la vacuna es segura.

- Creer que se posee un alto riesgo de infectarse de COVID 19.

Se obtuvieron como resultados, expresadas en opciones múltiples del tipo Escala Likert, en cada uno de los items:

Conveniencia de recibir la vacuna en términos de aplicación, cercanía y gratuidad: En gran parte $n=870$, Algo $n=830$, Muy poco $n=970$, Para nada $n=620$, No sé $n=40$. 


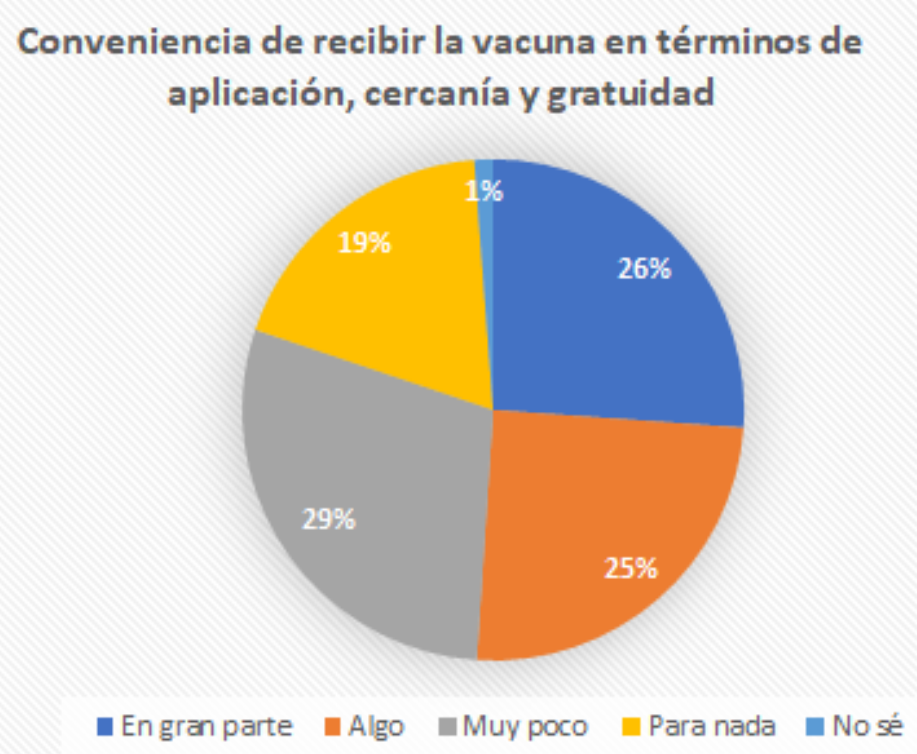

Recibir la recomendación de mi empleador y/o autoridad laboral para vacunarme: En gran parte $n=280$, Algo $n=490$, Muy poco $n=1130$, Para nada $n=1370$, No sé $n=60$.

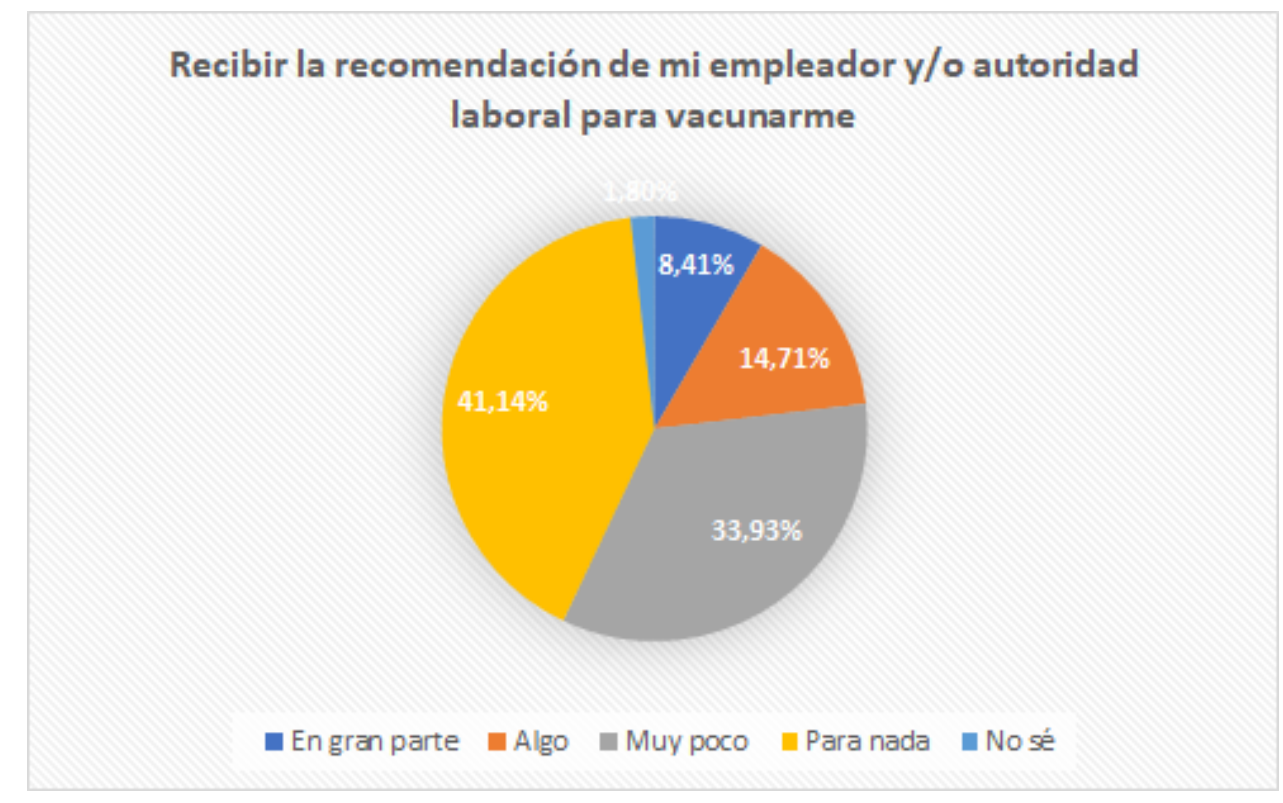

Tener información de que la vacuna es segura: En gran parte $n=2500$, Algo $n=560$, Muy poco $n=150$, Para nada $n=90$, No sé $n=30$. 


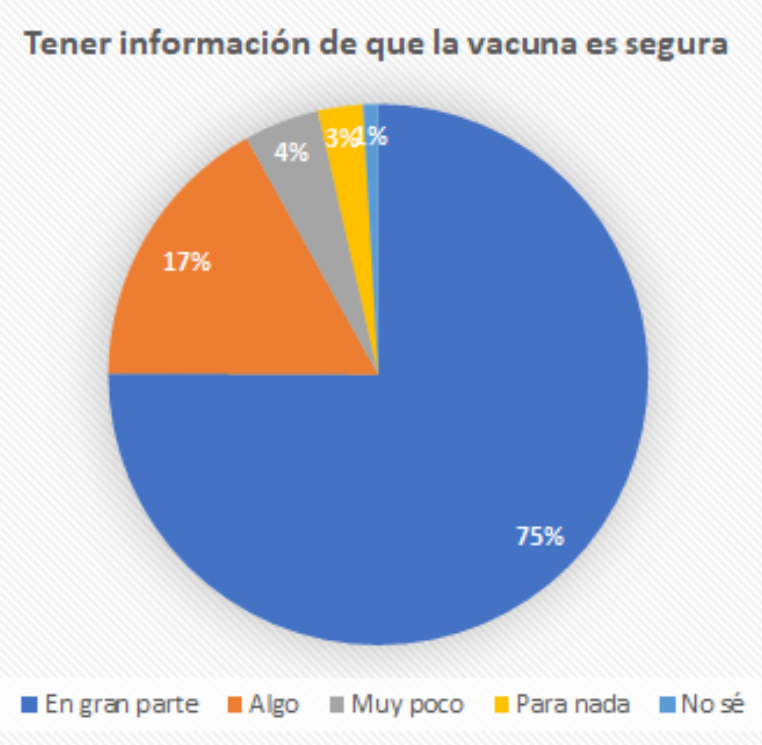

Creer que se posee un alto riesgo de infectarse de COVID 19: En gran parte $n=1640$, Algo $n=850$, Muy poco $n=510$, Para nada $n=300$, No sé $\mathrm{n}=30$.

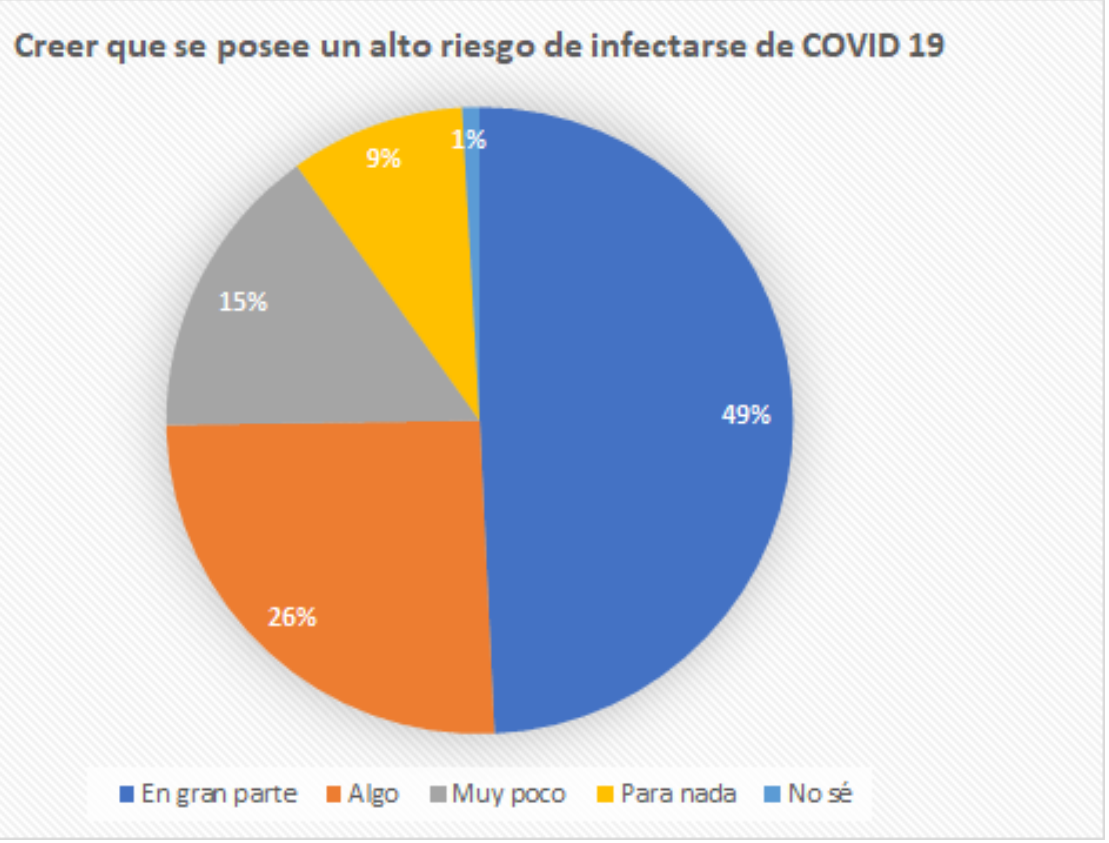

Al consultársele en qué medida estima continuar utilizando mascarillas y distanciamiento social mientras que dure el COVID luego de recibir la vacuna las respuestas obtenidas arrojaron como resultados: La mayor parte del tiempo 84,4 \% $(n=2810)$, Algunas veces 10,5\% 
$(\mathrm{n}=350)$, Rara vez $0,9 \%(\mathrm{n}=30)$, Nunca $1,2 \% \quad(\mathrm{n}=40)$ y No pienso vacunarme contra el COVID $194,2 \%(n=140)$.

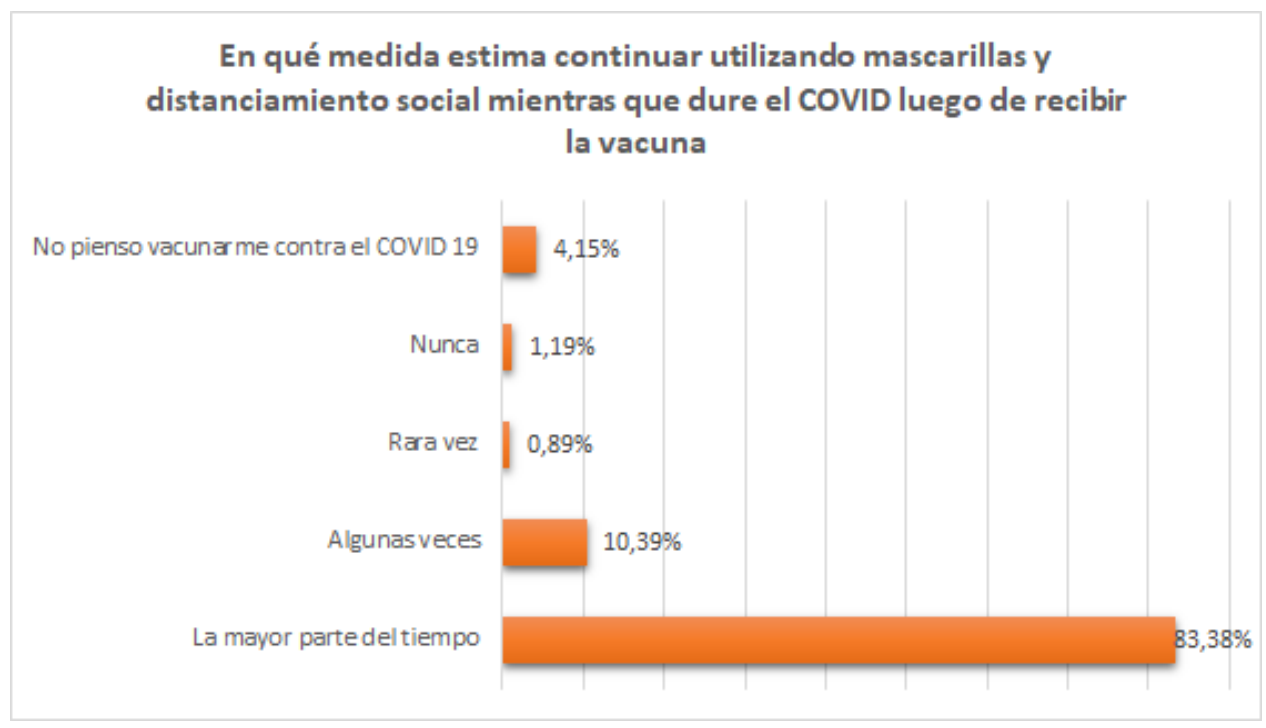

La pregunta acerca de si cambio su concepción sobre alguna de las vacunas entre el mes de enero y el día de completar la encuesta aporto como resultantes: Si 35,4\%(n=1180), No 52,3\% (n=1740) y Tal vez 13,2\% $(n=440)$.

\section{Cambio su concepción sobre alguna de las vacunas entre el mes de enero y el día de completar la encuesta}

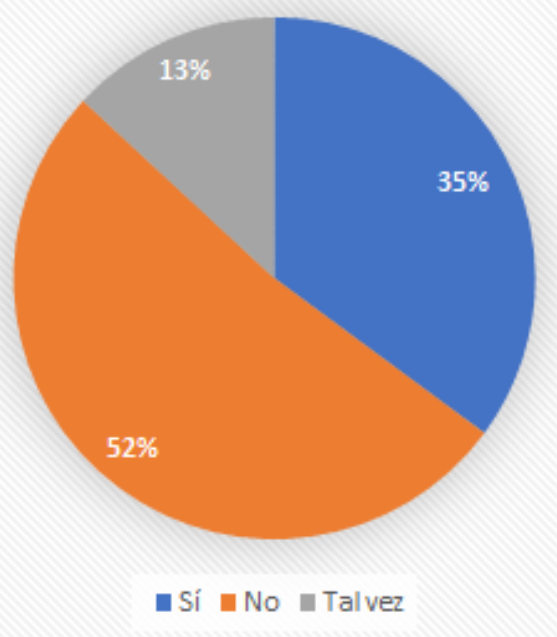

Le han sido brindadas una serie de opciones consultándosele si han incidido en su concepción acerca de las vacunas, a saber:

-Publicaciones científicas que presentaron resultados de eficacia $64,3 \%(n=2140)$. 
-Ver resultados en personas conocidas o allegadas 16,5\% ( $\mathrm{n}=550)$.

- Aceptación de los medios de comunicación y redes sociales de los resultados y emisión de comunicaciones positivas respecto de la vacuna y la vacunación 9,6\% $(\mathrm{n}=320)$.

El resto de los participantes presentaron opciones menores al 1\%, incluso la recomendación médica se seleccionó como motivación en un $0,9 \%(n=40)$.

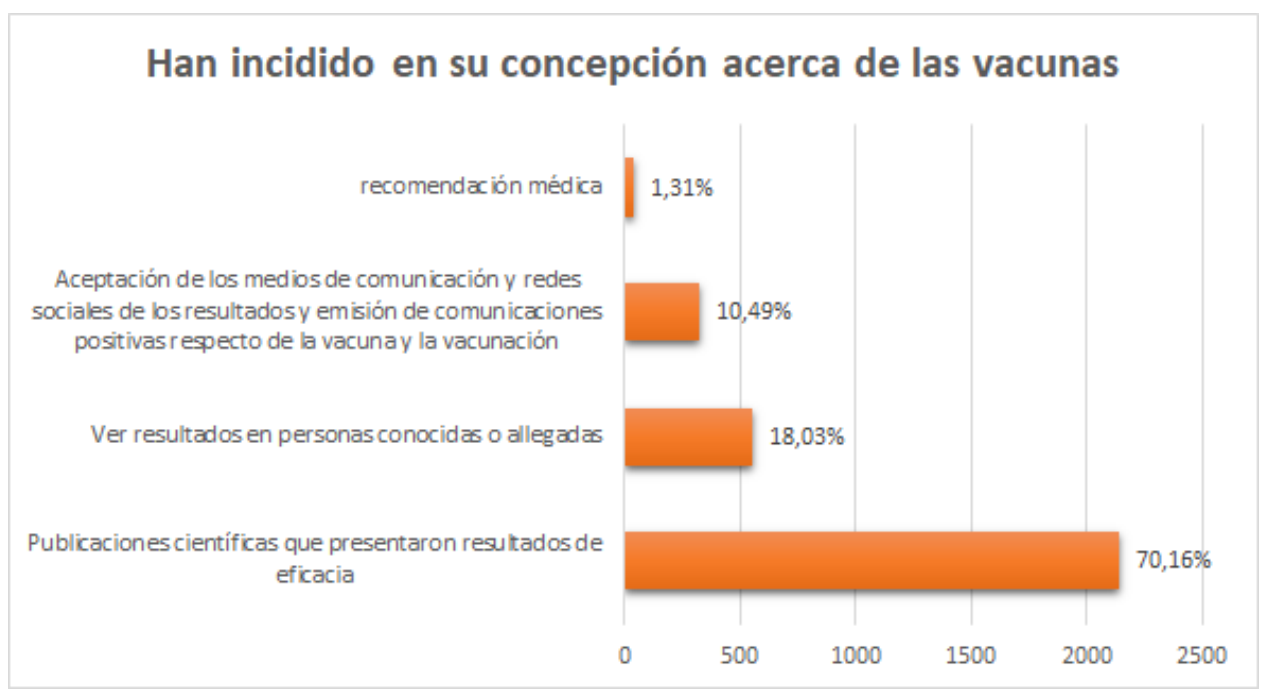

\section{Discusión}

Las representaciones se postulan como un estilo específico de conocimiento, un modo determinado de descifrar la realidad, aludiendo al pensamiento del sentido común, respondiendo a un desarrollo personal que supone una ordenación cognitiva y a la vez halla su inicio, su difusión y cambio en el contexto sociocultural.

Al momento del estudio el $48,9 \%$ se encontraba vacunado sin diferencias significativas al serle consultado si influencio en la conveniencia de recibir la vacuna en términos de aplicación, la cercanía y la gratuidad. En idéntico sentido en la mayoría de los participantes reconoce que la sugerencia o indicación de las autoridades y/o 
empleadores no fueron determinantes a la hora de vacunarse frente al COVID 19.

Los determinantes más influyentes para la vacunación han sido el contar con información acerca de la seguridad de la vacuna y el estimar considerarse en riesgo de contraer el COVID 19.

Otro dato de relevancia obtenido ha sido que mientras que persis ta el COVID 19 en circulación la gran mayoría de los encuestados estima que continuará utilizando mascarillas y distanciamiento social más allá de recibir de recibir la vacuna. Por el contrario 1 de cada cinco participantes expreso una negativa a vacunarse contra el COVID 19.

Siendo uno de los datos más reveladores que solo el 1,31\% de recomendación contribuyo en su concepción acerca de las vacunas y la vacunación, y por el contrario 7 de cada 10 se vio significativamente influenciado por las publicaciones científicas difundidas en torno a la eficacia de las vacunas. Estos datos revelan el poder actual de la comunicación en la ciencia y el grado de impacto no solo en la comunidad científica sin en la población general. Este dato debiera ser ponderado en grupos sociales de estratos más bajos con menor acceso al debate científico.

Las representaciones sociales de los participantes muestran una gran adherencia a la vacunación y a las vacunas, con conceptos positivos en 8 de cada de 10 y un claro sostenimiento de las medidas de protección de como aislamiento y uso de más carilla acompañando la inoculación mientras que se conviva con el COVID 19.

\section{Conclusiones}

La investigación es fundamental para el avance de la ciencia y más en esta época, en emergencia, donde es necesario disminuir la 
incertidumbre y evaluar las propuestas de vacunas y estrategias que permitan el control de la pandemia que, desde el año 2020, viene azotando al planeta. Desde la Salud Pública se ha venido trabajando arduamente para controlar y erradicar enfermedades a lo largo de la historia. De este modo, la poliomielitis, la tuberculosis, el sarampión, como tantas otras fueron eliminadas gracias a la existencia de vacunas que contribuyeron al control y erradicación de enfermedades como las citadas y otras más. Hoy en día, las vacunas disponibles para el control de la pandemia constituyen una muestra más del trabajo que viene desarrollando la ciencia al servicio de la humanidad. El mundo se vio amenazado pero la vigilancia en Salud y los estudios constantes permiten un mayor conocimiento y defensa frente al virus.

Las vacunas previas en la historia no se colocaron en primera línea de debate acerca de su procedencia y eficacia. El acceso a la comunicación inmediata, a las publicaciones científicas y a redes sociales permiten un extenso debate y someten a la opinión pública el avance de la ciencia frente al COVID 19 en simultáneo a que transcurre. El desafío es la superación del COVID 19 con el compromiso de todos más allá de las creencias y representaciones de cada uno.

(1)Autores:

Valeria Fernanda Altamirano

Universidad de Flores, Argentina.valeriaaltamirano33@hotmail.com

\section{Simon L. Bacon}

Montreal Behavioural Medicine Centre, CIUSSS du Nord-de-1'Ile-de-Montreal, Montreal, Québec, Canada.simon.bacon@concordia.ca

\section{Silvana Baró}

Universidad de Flores, Argentina.silvana_baro@yahoo.com.ar 


\section{Daniela Anahí Benitez}

Universidad de Flores, Argentina.d.a.benitez@hotmail.com.ar

\section{José Carlos Caravello}

Universidad Nacional de Lomas de Zamora, Argentina.

carloscarave1lo9@gmail.com

\section{Natalia Lucia Filippa}

Universidad de Flores, Argentina.nnatalia_filippa@hotmail.com

\section{Zulma Gabriela Gastaldo}

Universidad de Flores, Argentina.zgastaldo@gmail.com

\section{Carolina Gómez Jiménez}

Universidad de Flores, Argentina. carolinakph604@gmail.com

\section{Nora Estela Grañana}

Universidad de Flores, Argentina.ngranana@gmail.com

\section{Kim L. Lavoie}

Montreal Behavioural Medicine Centre, CIUSSS du Nord-de-1'Ile-de-Montreal, Montreal, Québec, Canada. kim.lavoie@mbmc-cmcm.ca

\section{Analia Verónica Losada}

Universidad de Flores, Argentina. analia_losada@yahoo.com.ar

\section{Patricia Inés Medina}

Universidad de Flores, Argentina. patriciai.medina@gmail.com

\section{Mónica Andrea Muñoz}

Universidad de Flores, Argentina. carpedoemmoni16@gmail.com

\section{Adilton Cicero Nogueira Da Silva}

Pontificia Universidad Católica Argentina, Argentina. ciceronogueira@uca.edu.ar

\section{Sandra Peláez}

Universidad de Montreal, Canadá.sandra.pelaez@umontreal.ca 


\section{Alicia Romina Pérez}

Universidad de Flores, Argentina.romina_trabajosocial@yahoo.com.ar

\section{Micaela Victoria Potes}

Universidad de Flores, Argentina.micaelapotes@hotmail.com

\section{Lucila Belén Santojanni}

Universidad de Flores, Argentina.1bsantojanni@gmail.com

\section{Sandra Estela Sorbara}

Universidad de Flores, Argentina.sandra.sorbara@uflo.edu.ar

\section{Selediana Souza Godinho}

Universidad de Flores, Argentina.selegodinho@gmail.com

\section{Alicia Etelvina Taliercio}

Universidad Nacional de Lomas de Zamora, Argentina. taliercio_juez@hotmail.com

\section{Romina Jacqueline Zaracho}

Universidad de Flores, Argentina. jacquezaracho24@gmail.com 


\section{Referencias}

Bacon, S. L., Baro, S., Barreira, I., Caravello, J. C., Caruso, J. M, Filippa, N. L., Gastaldo, Z. G., Gómez Jiménez, C., Grañana, N., Lavoiex, K. L., Losada, A. V., Muñoz, M. A., Nogueira Da Silva, A. C., Peláez, S., Peralta, M. C., Peralta, M. F., Pérez A. R., Pérez, A. R., Roma, M. C., Sorbara, S. E., Taliercio, A. E y De Souza Godinho, S. (2020). Representaciones de las medidas gubernamentales en el contexto del COVID19. Revista Digital prospectivas en Psicología, 5 (1), 15-24.

Losada, A. V. (2014). Uso en Investigación y Psicoterapia del Consentimiento Informado. En Kerman, B \& Rodriguez Ceberio, M. E. En búsque da de las ciencias de la mente. Editorial Universidad de Flores.

Ley 27573 (2020). Ley de Vacunas destinadas a generar inmunidad adquirida contra el Covid-19. http://servicios.infoleg.gob.ar/infole gInternet/verNorma.do?id=343958

Ministerio de Salud (2019). Plan Estratégico Para La Vacunación Contra la Covid-19 en la República Argentina. https://www.argentina.gob.ar/sites/default/files/coronavirus-vacuna-planestrategico-vacunacion-covid-19-diciembre-2020.pdf

Ministerio de Salud (2021). ¿Cuáles vacunas estamos aplicando en el país? https://www.argentina.gob.ar/coronavirus/vacuna/cuales

Ministerio de Salud (2021). Monitor Público de Vacunación. https://www.argentina.gob.ar/coronavirus/vacuna/aplicadas

Rivela, C., \& Losada, A. (2021). Representaciones Sociales en Organizaciones de Seguridad. Rol e Influencia en el Sentido de Vida. Revista Científica Arbitrada de la Fundación MenteClara. Vol. 6 (218). doi: https://doi.org/10.32351/rca.v6.218

Télam (2021). Falso. La vacuna de Pfizer ocasiono la muerte de seis personas tras su aplicación. https://confiar.telam.com.ar/falso-la-vacuna-de-pfizer-ocasiono-la-muerte-de-seis-personastras-su-aplicacion/

Télam (2021). Falso: las vacunas no sirven si no tienen más de190\% de eficacia. https://confiar.telam.com.ar/falso-las-vacunas-no-sirven-si-no-tienen-mas-del-90-de$\underline{\text { eficacia/ }}$ 